\title{
Spin gases as microscopic models for non-Markovian decoherence
}

\author{
L. Hartmann, ${ }^{1}$ J. Calsamiglia, ${ }^{1,3}$ W. Dür, ${ }^{1,2}$ and H.-J. Briegel ${ }^{1,2}$ \\ ${ }^{1}$ Institut für Theoretische Physik, Universität Innsbruck, Technikerstraße 25, A-6020 Innsbruck, Austria \\ ${ }^{2}$ Institut für Quantenoptik und Quanteninformation der Österreichischen Akademie der Wissenschaften, Innsbruck, Austria \\ ${ }^{3}$ Grup de Física Teòrica \& IFAE, Universitat Autònoma de Barcelona, 08193 Bellaterra, Barcelona, Spain
}

(Received 24 June 2005; published 10 November 2005)

\begin{abstract}
We analyze a microscopic decoherence model in which the total system is described as a spin gas. A spin gas consists of $N$ classically moving particles with additional, interacting quantum degrees of freedom (e.g., spins). For various multipartite entangled probe states, we analyze the decoherence induced by interactions between the probe and environmental spins in such spin gases. We can treat mesoscopic environments $\left(\approx 10^{5}\right.$ particles). We present results for a lattice gas, which could be realized by neutral atoms hopping in an optical lattice, and show the effects of non-Markovian and correlated noise, as well as finite-size effects.
\end{abstract}

DOI: 10.1103/PhysRevA.72.052107

PACS number(s): 03.65.Yz, 03.65.Ud, 03.67.Mn

Decoherence is a fundamental mechanism believed to be responsible for the transition from the quantum to the classical world [1]. Interactions between system degrees of freedom and (uncontrollable) environment degrees of freedom lead to entanglement, manifesting itself in the decoherence of the system state. Many decoherence models have been discussed in the literature, most prominent among them oscillator bath models [2] and spin bath models [3]. Here, we study a physically motivated model of a mesoscopic inhomogeneous spin bath. In particular, we describe the environment by a spin gas [4]. A spin gas is a system of quantum spins with stochastic time-dependent interactions. A physical model of a spin gas is a system of $N$ classically moving particles with additional, internal spin degrees of freedom. Upon collision, these quantum degrees of freedom interact according to some specified Hamiltonian. Hence, in such spin gases, classical kinematics drives the evolution of the quantum state, and also the decoherence of probe systems. In general, multiple nonconsecutive collisions of particles are possible. In this sense, a spin gas provides a microscopic model for non-Markovian decoherence.

In this paper, we determine an effective, time-dependent map that describes the decoherence process in a spin gas. For specific system-environment interactions, we can treat mesoscopic environments $\left(\approx 10^{5}\right.$ particles $)$ exactly and efficiently. To derive the map, we extend the description of certain states in terms of valence bond solids (VBSs) [5] to completely positive maps. We do not restrict the analysis to single-qubit probes, but also consider the effect of decoherence on various (multipartite) entangled probe states (cf. Ref. [6]). Due to the stochastic nature of the interactions, our model does not display symmetries, which otherwise simplify the treatment (see, e.g., Ref. [8] for homogeneous system-environment interactions). Throughout the paper, we concentrate on a specific realization of a spin gas, the spin lattice gas. However, our methods can be easily applied to decoherence in other spin gases, such as a Boltzmann gas [4]. Spin gases are not only toy models of theoretical interest, but could be experimentally realized with present-day technology and existing setups [7].

Model. The probe system $A$ consists of $N_{A}$ qubits prepared in some arbitrary state. The qubits of system $A$ interact with uncontrollable degrees of freedom of an environment $B$, leading to decoherence. We consider a microscopic decoherence model where the environment is described by a spin gas. All particles interact according to the time-dependent Hamiltonian

$$
H(t)=\sum_{k<l} g\left[\boldsymbol{r}_{k}(t), \boldsymbol{r}_{l}(t)\right] H_{k l}
$$

but we always put $g$ equal to zero if both $k$ and $l$ are system particles (other cases can but will not be considered). The function $g$ depends on the physical nature of the pairwise spin interaction described by the Hamiltonian $H_{k l}$. For such systems, we have shown $[4,9]$ that one can efficiently compute reduced density operators of up to ten particles even for mesoscopic system sizes $\left(\approx 10^{5}\right.$ particles), if all Hamiltonians $H_{k l}$ commute and the initial state of the system is a pure product state. Here, we extend these results to take arbitrary initial system states and mixed environmental states into account, and thereby study the decoherence of multipartite probe states in a mesoscopic environment efficiently and exactly.

For commuting Hamiltonians $H_{k l}$, the joint state of system and environment at a time $t,\left|\Psi_{t}\right\rangle=\prod_{k, l} e^{-i \varphi_{k l}(t) H_{k l}}\left|\Psi_{0}\right\rangle$, is determined by the interaction phases $\varphi_{k l}(t)$ $\equiv \int_{0}^{t} g\left[\boldsymbol{x}_{k}\left(t^{\prime}\right), \boldsymbol{x}_{l}\left(t^{\prime}\right)\right] d t^{\prime}$. These phases are associated with the adjacency matrix $\Gamma(t)$ of a weighted graph $G$. The matrix element $\Gamma_{k l}(t)=\varphi_{k l}(t)$ describes the neighborhood relation of particles $k$ and $l$, or, equivalently, the interaction history. We are interested in the state of system $A$, i.e., the reduced density operator $\rho_{A}(t)=\operatorname{tr}_{B}\left|\Psi_{t}\right\rangle\left\langle\Psi_{t}\right|$. The commutation of the interaction Hamiltonians greatly simplifies the computation of this operator: We have to take into account only interactions between particles $k \in A$ and $l \in B$, since interactions within $B$ do not change the state of system $A$. In contrast to the general case of noncommuting Hamiltonians [10], entanglement in the environment does not influence the decoherence properties of the system.

In the following, we treat the case $H_{k l}=|11\rangle_{k l}\langle 11|$ and initially completely polarized environment spins, $|\Psi\rangle_{B}=|+\rangle^{\otimes N}$ where $| \pm\rangle \propto|0\rangle \pm|1\rangle$. Extensions to other commuting interac- 
tion Hamiltonians and arbitrary (possibly mixed) product environmental states are straightforward. The isomorphism between completely positive maps and mixed states [11] together with a generalized valence bond solids (VBSs) picture [5,9] — determines the effective map $\mathcal{E}_{t}$ that takes an initial state at $t_{0}=0$ to the state at time $t$, i.e., $\rho_{A}(t)=\mathcal{E}_{t} \rho_{A}(0)$. This map $\mathcal{E}_{t}$ can be equivalently described by the state $E_{t}$ $=\mathbb{1}^{A^{\prime}} \otimes \mathcal{E}_{t}^{A}|\Phi\rangle\langle\Phi|$. Here, $|\Phi\rangle=\otimes_{k=1}^{|A|}\left|\phi^{+}\right\rangle_{k^{\prime} k},\left|\phi^{+}\right\rangle \propto|00\rangle+|11\rangle$, and $A^{\prime}$ is an auxiliary system with the same dimension as $A$. We can express the map in the Pauli basis $\sigma_{k}$, where $\sigma_{0} \equiv 1$. Then, $\quad \mathcal{E}_{t} \rho=\sum \lambda_{k_{1} \cdots k_{N_{A}}, l_{1} \cdots l_{N_{A}}} \sigma_{k_{1}} \cdots \sigma_{k_{N_{A}}} \rho \sigma_{l_{1}} \cdots \sigma_{l_{N_{A}}}, \quad$ where $\lambda_{k_{1} \cdots k_{N_{A}}, l_{1} \cdots l_{N_{A}}}=\left\langle\phi_{k_{1} \cdots k_{N_{A}}}\left|E_{t}\right| \phi_{l_{1} \cdots l_{N_{A}}}\right\rangle \quad$ with $\quad\left|\phi_{k_{1} \cdots k_{N_{A}}}\right\rangle=\mathbb{1}^{A^{\prime}}$ $\otimes\left(\sigma_{k_{1}}{ }^{\cdots} \sigma_{k_{N_{A}}}\right)^{A}|\Phi\rangle$. The coefficients of the map are given by the coefficients of the state $E_{t}$ written in (tensor products of) Bell bases. As in the case of states [9], we can separately consider maps (or equivalently the states $E_{t}^{(l)}$ ) resulting from the interaction of the system with a single particle $l$ in the environment. We find $E_{t}^{(l)}=1 / 2\left(|\Phi\rangle\left\langle\Phi\left|+\otimes_{k=1}^{|A|}\right| \chi_{k}\right\rangle\left\langle\chi_{k}\right|\right)$ with $\left|\chi_{k}\right\rangle_{k^{\prime} k}=1 / \sqrt{2}\left(|00\rangle+e^{-i \varphi_{k l}(t)}|11\rangle\right)$, where $\varphi_{k l}(t)$ is the effective interaction phase between particles $k \in A$ and $l \in B$. The state $E_{t}$ describing the total decoherence process incorporates the influence of all particles $l \in B$. We obtain $E_{t}$ (up to normalization) by calculating the Hadamard product of all $E_{t}^{(l)}$ written in the standard basis, i.e., by component-wise multiplication. The matrix elements of $E_{t}$ expressed in the tensor Bell basis finally determine $\mathcal{E}_{t}$. We find that $E_{t}$ has nonzero components only in the subspace spanned by $\left\{\left|\phi_{k_{1} \cdots k_{N_{A}}}\right\rangle\right\}$ with $k_{j} \in\{0,3\}$. The map $\mathcal{E}$ thus contains only tensor products of Pauli operators 1 and $\sigma_{z}$. Equivalently, we can express the action of the map on an arbitrary probe input state $\rho$ $=\Sigma_{s, s^{\prime}} \rho_{\mathrm{ss}^{\prime}}(0)|\mathbf{s}\rangle\left\langle\mathbf{s}^{\prime}\right|$ by determining the evolution of the coherences $\rho_{\mathrm{ss}^{\prime}}(t)$. With $\mathbf{s}_{A}, \mathbf{s}_{A}^{\prime}$ we denote binary vectors of length $N_{A}$. We can express the coherences as $\rho_{\mathrm{ss}^{\prime}}(t)$ $=C_{\text {ss }^{\prime}}(t) \rho_{\text {ss }^{\prime}}(0)$ with [4]

$$
C_{\mathbf{s} \mathbf{s}^{\prime}}(t)=e^{i(1 / 2) \Sigma_{k}\left(\mathbf{s}_{A}-\mathbf{s}_{A}^{\prime}\right) \cdot \Gamma_{\mathbf{k}}} \prod_{k=1}^{N_{B}} \cos \left[\frac{1}{2}\left(\mathbf{s}_{A}-\mathbf{s}_{A}^{\prime}\right) \cdot \boldsymbol{\Gamma}_{\mathbf{k}}\right] .
$$

The $\left(\boldsymbol{\Gamma}_{\mathbf{k}}\right)_{j}=\Gamma_{k j}$ for each particle $k \in B$ are $N_{A}$-dimensional vectors. The method described above can be considered as an extension of the generalized VBS picture for states to one for completely positive maps. In this picture, we can determine the evolution of arbitrary system states in a mesoscopic spin environment, since the computational effort to calculate the maps scales only linearly with the number of particles in the environment (as opposed to exponentially for general system-environment interactions). The size of the probe system is limited to about ten spins for numerical computations due to exponential scaling with system size $N_{A}$.

The quantum properties of the system are directly linked to the classical statistical properties of the gas through $\Gamma(t)$. In general, it is thus necessary to know the classical $n$-body phase-space distributions to give a complete description of the quantum state. If we assume no control over quantum or classical degrees of freedom of the background gas we should average over all possible collision patterns at any given time [4]: $\bar{C}_{s, s^{\prime}}(t)=\int d \Gamma p_{t}(\Gamma) C_{s, s^{\prime}}(\Gamma)$, where $p_{t}(\Gamma)$ is the probability that at time $t$ the interaction history is given by $\Gamma$.

For some gas models and regimes (like the Boltzmann gas studied in Ref. [4]), correlations play a minor role and one can find analytical expressions for single-particle phasespace distributions. In this paper, however, we study a lattice gas model that exhibits strong correlations, and produce the different random realizations of $\Gamma(t)$ by direct simulation of the gas.

The lattice model can be possibly implemented in a quantum optical system. It has already been demonstrated that an optical lattice can be used to store ultracold atomic gases. The degree of control in these experiments is extraordinary, opening the door to a wide range of experiments and theoretical proposals [12-18]. One can choose a parameter regime where each lattice site is occupied by at most one atom $[12,15]$. The internal state of the atom (e.g., two metastable hyperfine states) can be stored in coherent superpositions over long time scales (few minutes). Coherent interatomic interactions have been achieved by cold collisions [13]. These correspond precisely to the Ising-type interactions chosen here. One can also find schemes [8] to induce a random (incoherent) hopping of atoms from one site to its neighboring sites. Hence we consider an $M \times M$ lattice containing $N$ particles that randomly hop from site to site with a hopping rate $\eta$. These particles interact whenever they are nearest neighbors, i.e., we put $g\left[\boldsymbol{r}_{k}(t), \boldsymbol{r}_{l}(t)\right]$ from Eq. (1) equal to some constant $g_{0}$ whenever particle $k$ and $l$ are nearest neighbors and to zero otherwise. With special relevance to possible experiments, we note that all results in this paper hold even when the environment particles themselves decohere. The only requirement is that the diagonal elements of the environment's state-in the canonical basis-remain unchanged.

Decoherence of a single qubit. For a system consisting of a single qubit $A=\{1\}$ and an arbitrary environment, the timedependent map corresponding to a particular collisional history is

$$
\mathcal{E}_{t} \rho=\lambda_{00} \rho+\lambda_{11} \sigma_{z} \rho \sigma_{z}+\lambda_{01}\left(\rrbracket \rho \sigma_{z}-\sigma_{z} \rho \rrbracket\right),
$$

with $\quad \lambda_{00}=(1+r \cos \gamma) / 2, \quad \lambda_{11}=(1-r \cos \gamma) / 2, \quad$ and $\quad \lambda_{01}$ $=(i r \sin \gamma) / 2 \quad$ where $\quad r(t)=\prod_{l \in B} \cos \left(\varphi_{1 l}(t) / 2\right), \gamma(t)$ $=\Sigma_{l \in B}\left(\varphi_{1 l} / 2\right)$. Depending on the parameter regime, semiquantal gases can follow various collision patterns. Accordingly, the dynamics of their quantum properties can differ considerably. If in every time step $\delta t$ a given particle collides with a different particle and acquires an interaction phase $\delta_{\varphi}$, the dynamics will be purely Markovian. The coherence of that particle will decay exponentially fast with the number of time steps $k=\Delta t / \delta t,\left|\rho_{01}\right|=\left[\cos \left(\delta_{\varphi} / 2\right)\right]^{k}=e^{-\Delta t / \tau_{e}}$ with $\tau_{e}$ $\approx 8 \delta t / \delta_{\varphi}^{2}$. If, on the other hand, in a small time interval $\Delta t$ a given gas particle has collided $k$ times with the same particle, the coherent addition of the interaction phase leads to a Gaussian type of decay: $\left|\rho_{01}\right|=\cos \left(k \delta_{\varphi} / 2\right) \approx e^{-\Delta t^{2} /\left(2 \tau_{g}^{2}\right)}$ with $\tau_{g}=2 \delta t / \delta_{\varphi}$. The exponential and the Gaussian decay are the two extreme cases, the dynamics of the coherence $\left|\rho_{01}\right|$ will usually lie in between. This also holds for the coherences in a multiqubit density matrix. A complete characterization of the system's decoherence is obtained by averaging the maps 

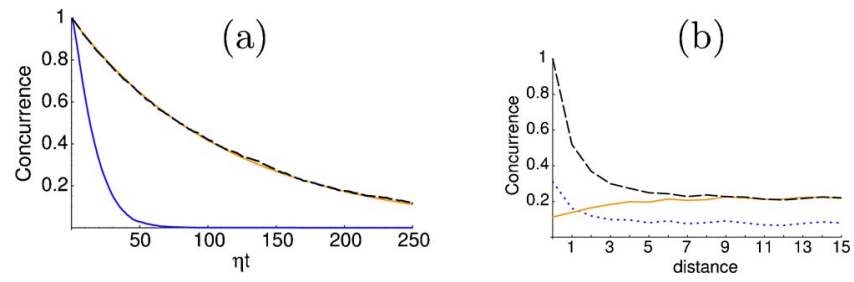

FIG. 1. (Color online) (a) Concurrence of two probe particles prepared in a maximally entangled Bell state $\left|\phi^{+}\right\rangle \propto|00\rangle+|11\rangle$ in a $100 \times 3200$ lattice gas with $N=8 \times 10^{4}$ for $g_{o}=0.8 \eta$ and fixed probes (solid ); and fast moving probes (dashed). The solid, light curve corresponds to an analytical result obtained in a Markovian regime. (b) Concurrence of two probe particles at time $t_{1}=25 \eta^{-1}$ for different initial entangled states as a function of distance $d$ between the probes: $\left|\psi^{+}\right\rangle \propto|01\rangle+|10\rangle$ (dashed-dark), $\left|\phi^{+}\right\rangle$(solid-light), and $|G\rangle \propto|+0\rangle+|-1\rangle$ (dotted).

$\mathcal{E}_{t}$ over the possible collision patterns. The resulting map has the same form as Eq. (3), but replacing the coefficients $\lambda_{i j}$ by their average values. An analytical expression for the time dependence of these averaged coefficients, and hence of the decoherence process, is in general hard to obtain. However, for finite lattices, one can give a precise description of the time dependence.

Decoherence of bipartite entangled states. We now study the decoherence of different, initially entangled two-qubit states. In order to explore different regimes, we imagine a scenario where the probe particles can be displaced at a constant speed $v$ relative to the gas. By varying the speed $v$ and the distance $d$ between the probe particles, we can highlight two effects: (i) By decreasing the probe speed, we analyze the effect of multiple interactions with the same particle in contrast to interactions with different (independent) particles. (ii) By increasing the distance, we turn from correlated to independent collisions between probe and environment particles.

Figure 1(a) shows the decay of entanglement, measured by the concurrence [19], of an initial Bell state $\left|\phi^{+}\right\rangle$in two extreme scenarios: (i) fixed probe particles $(v=0)$; (ii) large probe speeds, $v / a \gg \eta$, where $a$ is the intersite spacing. A fixed value $\varphi=0.1$ is assigned to the collisional phase every time a probe particle crosses an occupied site. These two scenarios illustrate the difference between Markovian and non-Markovian environments. A large probe speed enforces a perfect Markovian behavior which matches the analytical curve [20]. Figure 1(b) shows the concurrence at a given time $t_{1}$ as a function of the distance for three different entangled states: two Bell states and a cluster state (see figure caption). For Bell states the concurrence is equal to the absolute value of their only nonzero off-diagonal element in the density matrix, and therefore Fig. 1 provides direct information about the individual coherences. The figure shows the influence of correlated collisions: coherences $\rho_{01,10}\left(\right.$ in $\left|\psi^{+}\right\rangle$) are robust against correlated noise, and coherences $\rho_{00,11}$ (in $\left.\left|\phi^{+}\right\rangle\right)$are especially fragile under correlated noise. The remaining coherences decay in the same way under correlated or uncorrelated noise (hence the weak distance dependence of $|G\rangle)$. From Fig. 1(b) we also see that the immediate environments of each probe become more independent as $d$ increases.
The different behavior under correlated and uncorrelated collisions can be readily understood. For two probe particles (1 and 2) with very similar collision patterns, i.e., $\Gamma_{2 j}=\Gamma_{1 j}$ $+\delta_{j}$ for all $j$, coherences associated with $|01\rangle\langle 10|$ will only

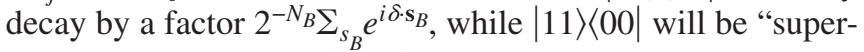

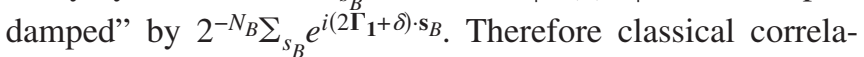
tions in the collisions, e.g., induced by the geometry of the setup, can significantly influence the entanglement properties of the system.

Decoherence of multipartite entangled states. We now apply our method to investigate the decoherence of different multipartite entangled probe states of $N_{A}$ qubits. Due to the lack of simple, computable multipartite entanglement measures, we use the negativity of bipartitions [21] as an indicator of multipartite entanglement in the system. That means that we consider bipartitions of the system, i.e., a partition consisting of a set of particles $A_{k}$ and its complement $\bar{A}_{k}$, and investigate the entanglement properties with respect to the $2^{N_{A}-1}-1$ independent bipartitions [22]. In general, we get a broad picture of multipartite entanglement in this way. For each bipartition we can determine its negativity $\mathcal{N}_{A_{k}}$ $=\left(\left\|\rho^{T_{A_{k}}}\right\|_{1}-1\right) / 2$ [14]. We define two multipartite entanglement measures: (i) the average negativity $\overline{\mathcal{N}}$, as the average over all bipartitions, $\overline{\mathcal{N}}=1 /\left(2^{\left(N_{A}-1\right)}-1\right) \Sigma_{A_{k}} \mathcal{N}_{A_{k}}$, and (ii) $\mathcal{N}_{\text {min }}=\min \left\{\mathcal{N}_{A_{k}}\right\}$. Zero average negativity is a necessary condition for full separability of the state, and $\mathcal{N}_{\min }=0$ is a sufficient condition that the state is not multiparty distillably entangled.

We have examined different multipartite entangled probe states that interact with a lattice gas through a pairwise Hamiltonian $H_{k l}=\sigma_{z}^{(k)} \otimes \sigma_{z}^{(l)}$ (chosen for convenience instead of the locally unitarily equivalent Hamiltonian $H_{k l}$ $\left.=|11\rangle_{k l}\langle 11|\right)$. These probe states are (linear) cluster states $|\chi\rangle$ [23], GHZ states and $W$ states $|W\rangle \propto \sum_{i=1}^{N_{A}}\left|w_{i}\right\rangle$, where $\left|w_{i}\right\rangle$ is the state corresponding to all spins in state zero except the $i$ th that is in one. Since the noise process is basis dependent, we study variants of GHZ states corresponding to different local bases: $\quad|\Psi\rangle \propto|0\rangle^{\otimes N_{A}}+|1\rangle^{\otimes N_{A}},\left|\Psi^{\prime}\right\rangle \propto|0\rangle|+\rangle^{\otimes N_{A}-1}$ $+|1\rangle|-\rangle^{\otimes N_{A}-1},\left|\Psi^{\prime \prime}\right\rangle \propto|+\rangle|0\rangle^{\otimes N_{A}-1}+|-\rangle|1\rangle^{\otimes N_{A}-1}$. The decay of the average negativity is plotted in Fig. 2(a). To qualitatively understand the different behavior of the curves we first derive analytic results in the limit of independent environments for each particle $k \in A$. In this limit, the decoherence process can be described by a tensor product of single-qubit dephasing maps $\mathcal{E}_{t}^{(k)} \rho=p_{k} \rho+\left(1-p_{k}\right) \sigma_{z}^{(k)} \rho \sigma_{z}^{(k)}$, where $p_{k}=\frac{1}{2}\{1$ $\left.+\prod_{l \in B} \cos \left[2 \varphi_{k l}(t)\right]\right\}$. Coherences decay as $\rho_{01}^{(k)}(t)=\left(2 p_{k}\right.$ $-1) \rho_{01}(0)$. As before, the precise time dependence of decoherence will be given by the average $p(t)=\left\langle p_{k}\right\rangle_{\Gamma(t)}$ over different realizations. We assume that this average value is the same for every probe particle $k$. Under the action of this dephasing map the family of GHZ states remain diagonal in the GHZ-type basis [22]. For such states, we can obtain the spectrum of the partial transposed operators with respect to any bipartition analytically [24], and calculate average negativities for the three GHZ states given above. Here we give those with simple expressions:

$$
\overline{\mathcal{N}}=\frac{1}{2}|2 p-1|^{N_{A}}
$$

and 

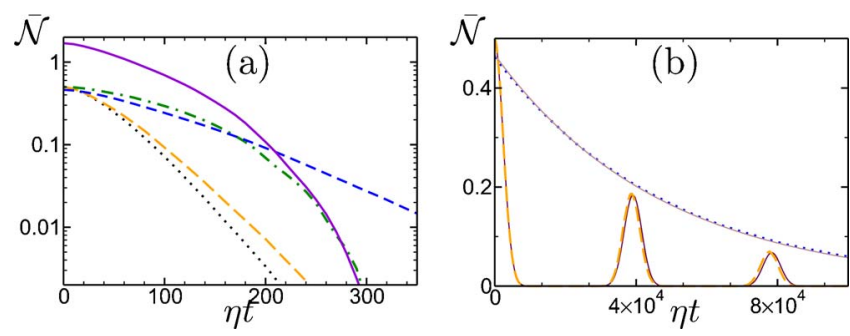

FIG. 2. (Color online) (a) Log-linear plot showing the decay of the average negativity $\overline{\mathcal{N}}$ for six-qubit linear cluster state (solid), $W$ state (short-dashed), and GHZ states $|\Psi\rangle$ (dotted), $\left|\Psi^{\prime}\right\rangle$ (dashdotted), and $\left|\Psi^{\prime \prime}\right\rangle$ (long-dashed) in a lattice gas with 400 spins in a $40 \times 40$ lattice and coupling constant $g_{0}=0.8 \eta$. (b) Entanglement decay of four-qubit GHZ (long-dashed) and $W$ (dotted) states in $20 \times 20$ lattice with 25 spins and $g_{0}=8 \times 10^{-3} \eta$ illustrating finitesize effects. Single-parameter $\left(\alpha^{\prime} s\right)$ analytical fits are also shown (thin lines).

$$
\overline{\mathcal{N}}^{\prime \prime}=\frac{1}{2^{N_{A}-1}-1}\left[\mathcal{N}_{\min }^{\prime \prime}+\left(2^{N_{A}-1}-2\right) \frac{1}{2}|2 p-1|^{N_{A}-1}\right]
$$

with

$$
\mathcal{N}_{\min }^{\prime \prime}=\frac{1}{2} \max \left\{0,|2 p-1|+|2 p-1|^{N_{A}-1}+|2 p-1|^{N_{A}-1}\right\} .
$$

For $W$ states, direct calculation leads to

$$
\overline{\mathcal{N}}^{W}=\frac{|2 p-1|^{2}}{N_{A}\left(2^{N_{A}}-2\right)} \sum_{a=1}^{N_{A}-1}\left(\begin{array}{c}
N_{A} \\
a
\end{array}\right) \sqrt{a\left(N_{A}-a\right)} .
$$

Several observations follow from these analytic results for independent environments: (i) Standard GHZ, $|\Psi\rangle$, and $W$ states remain $N_{A}$-party distillable for all times, since $\mathcal{N}_{\text {min }}$ only reaches zero asymptotically as $t \rightarrow \infty$. This does not hold for the $\left|\Psi^{\prime \prime}\right\rangle \mathrm{GHZ}$, which has one partition that becomes disentangled at finite $t$, nor for $\left|\Psi^{\prime}\right\rangle$, for which $\overline{\mathcal{N}}$ vanishes at a finite time (all partitions are disentangled). In Fig. 2(a) we see that $\overline{\mathcal{N}}$ also vanishes at finite time for the cluster state. (ii) In the limit of large system sizes, the average negativity is, to a very good approximation, given by the negativity of the half-half partition (the distribution of partitions with $k$ particles is sharply peaked at $k=N_{A} / 2$ ). (iii) GHZ-type states decay exponentially as we increase the system size $N_{A}$, while for $W$ the coherences do not vary with the system size, rendering a weak dependence of its average negativity (constant to first order). (iv) For the states studied here, if a partition is initially more entangled than another, it will remain so also at later times. This does not hold in the presence of correlated collisions, which occur when the distance between probe particles is not large with respect to the relevant times $t$, i.e., $d<\sqrt{\eta t}$.
In finite lattices and after long enough times the above description fails due to inevitable correlated collisions. However, the finite size leads to interesting effects that can also be easily understood. Figure 2(b) shows one such effect: the coherence (and hence the probe's entanglement), lost due to the stochastic interaction with environment spins, is partially recovered after a characteristic revival time. After long times a given environment particle will have collided $n_{k}$ times with a probe particle $k$. After $s \gg M^{2}$ steps, we describe the distribution of values $n_{k}$ by a Gaussian of mean value $\left\langle n_{k}\right\rangle=n$ $=4 s / M^{2}$ and the variance $\sigma^{2}=\left\langle\left(n_{k}-n\right)^{2}\right\rangle=\alpha n$, where $\alpha$ depends on the particular lattice model. We expect that at long times most blocking effects between environment particles will be washed out, and therefore assume that different environment particles will have independent collision distributions. Hence the total effect of $N_{B}$ on a particular coherence will scale as $C^{N_{B}}$, where $C$ is the decay factor of the coherence due to a single environment particle. The value of $C$ is given by an average taken over a Gaussian of mean $\varphi_{o}$ and width $\sigma_{\varphi}: C=\langle\cos (2 \varphi)\rangle=\cos \left(2 \varphi_{o}\right) \exp \left(-8 \sigma_{\varphi}^{2}\right)$. The phase $\varphi$ is a sum of the collisional phases (with the corresponding signs) involved in the particular coherence. For example, for a standard GHZ all phases are added $\varphi=\sum_{k=1}^{N_{A}} \varphi_{1 k}$ leading to a mean value $\varphi_{o} \approx N_{A} n \delta_{\varphi}$ and a variance $\sigma_{\varphi}^{2} \approx \alpha^{\prime} N_{A} n \delta_{\varphi}^{2}$ [25]. For the $W$ state and a given coherence, say $\rho_{w_{1} w_{2}}$, the phase is $\varphi=\varphi_{1 k}-\varphi_{2 k}$, leading to a vanishing mean value and to a purely exponential decay with $\sigma_{\varphi}^{2} \approx \alpha^{\prime \prime} 2 n \delta_{\varphi}^{2}$ - which is independent of the system size $N_{A}$. The exponential decay of the $W$ state and the periodic revival of the GHZ state can be clearly identified in Fig. 2(b).

Summary. We have studied a microscopic, exact model for non-Markovian decoherence where the joint system is described by a spin gas. Using a generalized VBS picture for maps, we have determined the time-dependent maps for the decoherence process. We studied the decay of entanglement for different multipartite entangled probe states in a lattice gas with a mesoscopic number of particles. Depending on the parameters of the gas, we have shown how to reach qualitatively different regimes such as Markovian- and nonMarkovian, and correlated and noncorrelated decoherence processes. For finite lattices we find that, although the interactions with the environment are stochastic, entanglement in the probes can spontaneously revive at a time given by the size of the lattice and independent of the number of environment particles.

We thank J. Asbóth for reading the manuscript. This work was supported by the FWF, the European Union (Grant Nos. IST-2001-38877, IST-2001-39227, OLAQUI, SCALA), the DFG, the ÖAW through Project APART (W.D.), and the Ramón y Cajal program (J.C.). 
[1] W. Zurek, Rev. Mod. Phys. 75, 715 (2003).

[2] A. Leggett et al., Rev. Mod. Phys. 59, 1 (1987).

[3] N. Prokof'ev and P. Stamp, Rep. Prog. Phys. 63, 669 (2000).

[4] J. Calsamiglia et al., quant-ph/0502017.

[5] F. Verstraete and J. I. Cirac, Phys. Rev. A 70, 060302(R) (2004).

[6] A. R. R. Carvalho, F. Mintert, and A. Buchleitner, Phys. Rev. Lett. 93, 230501 (2004).

[7] M. Ziman, P. Stelmachovic, and V. Buzek, Open Syst. Inf. Dyn. 12 (1) 81 (2005).

[8] Joint work with P. Zoller and M. Lewenstein (unpublished).

[9] W. Dür et al., Phys. Rev. Lett. 94, 097203 (2005).

[10] C. M. Dawson et al., Phys. Rev. A 71, 052321 (2005).

[11] J. I. Cirac et al., Phys. Rev. Lett. 86, 544 (2001).

[12] M. Greiner et al., Nature (London) 415, 39 (2002).

[13] O. Mandel et al., Nature (London) 425, 937 (2003).

[14] B. Paredes et al., Nature (London) 429, 277 (2004).

[15] D. Jaksch et al., Phys. Rev. Lett. 81, 3108 (1998).

[16] W. Hofstetter et al., Phys. Rev. Lett. 89, 220407 (2002).
[17] L.-M. Duan et al., Phys. Rev. Lett. 91, 090402 (2003).

[18] B. Damski et al., Phys. Rev. Lett. 91, 080403 (2003).

[19] W. K. Wootters, Phys. Rev. Lett. 80, 2245 (1998).

[20] At every time step, each probe particle interacts with a new environment particle with probability $\nu$. Hence, after a number $s$ of time steps, the relevant coherence is given by $\left|C_{00,11}\right|$ $=\left|C_{0,1}\right|^{2}=\left|\nu \exp \left(i \delta_{\varphi} / 2\right) \cos \left(\delta_{\varphi} / 2\right)+(1-\nu)\right|^{2 s}$.

[21] G. Vidal and R. F. Werner, Phys. Rev. A 65, 032314 (2002).

[22] W. Dür and J. I. Cirac, Phys. Rev. A 61, 042314 (2000); 62, 022302 (2000).

[23] H.-J. Briegel and R. Raussendorf, Phys. Rev. Lett. 86, 910 (2001).

[24] M. Hein, W. Dür, and H. J. Briegel, Phys. Rev. A 71, 032350 (2005); W. Dür and H. J. Briegel, Phys. Rev. Lett. 92, 180403 (2004).

[25] The factor $\alpha^{\prime}$ (and $\alpha^{\prime \prime}$ below) also includes the contribution of $K_{k k^{\prime}}=\left\langle n_{k^{\prime}} n_{k}\right\rangle-n^{2} \propto n$, which depends on the distance between the probe particles $k, k^{\prime}$. 